\title{
On the spectral dimension of random trees
}

\author{
Bergfinnur Durhuus 叫 Thordur Jonsson非 and John Wheater"非 \\ ${ }^{1}$ Department of Mathematics, University of Copenhagen, Universitetsparken 5, 2100 Copenhagen $\emptyset$ \\ ${ }^{2}$ University of Iceland, Dunhaga 3, 107 Reykjavik, Iceland \\ ${ }^{3}$ Rudolf Peierls Centre for Theoretical Physics, University of Oxford, 1 Keble Road, OX1 3NP, UK
}

\begin{abstract}
We determine the spectral dimensions of a variety of ensembles of infinite trees. Common to the ensembles considered is that sample trees have a distinguished infinite spine at whose vertices branches can be attached according to some probability distribution. In particular, we consider a family of ensembles of combs, whose branches are linear chains, with spectral dimensions varying continuously between 1 and $3 / 2$. We also introduce a class of ensembles of infinite trees, called generic random trees, which are obtained as limits of ensembles of finite trees conditioned to have fixed size $N$, as $N \rightarrow \infty$. Among these ensembles is the so-called uniform random tree. We show that generic random trees have spectral dimension $d_{s}=4 / 3$.
\end{abstract}

Keywords: spectral dimension, random combs, random trees

\section{Introduction}

Random walks on random geometric structures have received considerable attention in recent years, the motivation coming from a wide range of different areas of science. We mention here percolation theory [1], where the percolation clusters provide fluctuating geometries, diffusion in disordered media [4] and quantum gravity, where space-time itself is treated as a fluctuating manifold [3]. In particular, the long time characteristics of diffusion have been studied for the purpose of providing quantitative information on the mean large scale behaviour of the geometric objects in question. The spectral dimension is one example of such a quantity. In this article the geometric structures under consideration will be graphs with a distinguished vertex $r$, in which case the spectral dimension $d_{s}$ is defined by

$$
p_{t} \sim t^{-d_{s} / 2} \text { for } t \rightarrow \infty
$$

where $p_{t}$ denotes the return probability for the simple random walk starting at $r$ as a function of (discrete) time $t$, averaged with respect to the given probability distribution of graphs. (Below we reformulate this definition in terms of the generating function for the sequence $p_{t}, t \in \mathbb{N}$.)

The exact value of $d_{s}$ is known only for a rather limited class of models. In particular, for bond percolation on a hypercubic lattice the value of $d_{s}$ for the incipient infinite cluster at criticality is unknown, but is conjectured to be $4 / 3$ in sufficiently high dimensions [1]. And for planar maps related to two-dimensional quantum gravity it is likewise unknown, but conjectured on the basis of numerical simulations and scaling relations to be 2 [2]. For earlier work on random combs and random trees see [4, 6, 13] and references quoted there.

The main purpose of this article is to describe recent results and relevant parts of the methods employed in their proof, allowing a determination of the spectral dimension for a variety of ensembles of tree graphs. The methods also apply to other critical exponents, and some of them also have a wider scope of applicability which we hope to develop further in the future. The interested reader is referred to [8, 9] for further results and details.

We now introduce some basic definitions and notation. Let $G$ be a connected graph with a distinguished vertex $r$, called the root, and let $\sigma_{i}$ denote the degree (or valency) of a vertex $i$. We let $Q_{G}(x)$ denote the generating function for return probabilities of the simple random walk on $G$ defined by

$$
Q_{G}(x)=\sum_{\omega: r \rightarrow r} z^{|\omega|} \prod_{i \in \omega} \sigma_{i}^{-1}, \quad x=1-z^{2}, \quad 0<z<1 .
$$

\footnotetext{
†durhuus@math.ku.dk

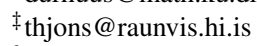

$\S_{\text {j.wheater@physics.ox.ac.uk }}$ 
where the sum is over all walks in $G$ starting and ending at the root $r$, including the trivial walk consisting of $r$ alone, $|\omega|$ denotes the number of links in $\omega$, and the product is over all vertices $i$ in $\omega$ counted separately for each visit of the walk except for the endpoint.

We then have the obvious identity

$$
Q_{G}(x)=\frac{1}{1-P_{G}(x)},
$$

where $P_{G}(x)$ is the generating function for first return probabilities given by the same formula as for $Q_{\tau}(x)$ except that the sum is restricted to walks that do not visit $r$ in between the initial and final position, and excluding the trivial walk.

Given a probability measure $\nu$ on a space $\mathcal{G}$ of connected graphs with a distinguished vertex $r$, we shall refer to $(\mathcal{G}, \nu)$ as an ensemble of graphs, and set

$$
Q(x)=\left\langle Q_{G}(x)\right\rangle_{\nu},
$$

where $\langle\cdot\rangle_{\nu}$ denotes the expectation w.r.t. $\nu$. If $Q(x)$ diverges as $x \rightarrow 0$, the spectral dimension of the ensemble is defined to be $d_{s}$, if for every $\epsilon>0$ there exist positive constants $\underline{c}_{\epsilon}, \bar{c}_{\epsilon}$ such that

$$
\underline{c}_{\epsilon} x^{\frac{d_{s}}{2}-1+\epsilon} \leq Q(x) \leq \bar{c}_{\epsilon} x^{\frac{d_{s}}{2}-1-\epsilon} .
$$

\section{Random combs}

In this section we determine the spectral dimension for some rather simple examples of random graphs, called random combs.

In order to define a comb, let $N_{\infty}$ denote the non-negative integers regarded as a graph with edges $(n, n+1), n=0,1,2, \ldots$, and similarly let, for a non-negative integer $\ell, N_{\ell}$ be the graph with vertices $\{0,1, \ldots, \ell\}$ and edges $(n, n+1), n=0,1, \ldots, \ell-1$. These are called linear graphs. A comb $C$ is a graph containing $N_{\infty}$ as a subgraph, called the spine (or backbone) of $C$, such that $C$ is obtained by attaching a copy $T_{n}$ of a linear graph $N_{\ell_{n}}$ at each vertex $n=1,2,3, \ldots$ on the spine except the first one, 0 , which will be called the root of the comb. Attaching $T_{n}$ at the vertex $n$ means here that the first vertex 0 in $T_{n}$ is identified with $n$ and otherwise no identifications are being made, see Fig. 1. $T_{n}$ will be called the $n$ 'th tooth of $C$ and $\ell_{n}$ its length, which may be finite or infinite. If $\ell_{n}=0$ we say that no tooth is attached at $n$. The set of all combs will be denoted by $\mathcal{C}$. For the comb $N_{\infty}$ without any teeth and the comb $N_{*}$ with

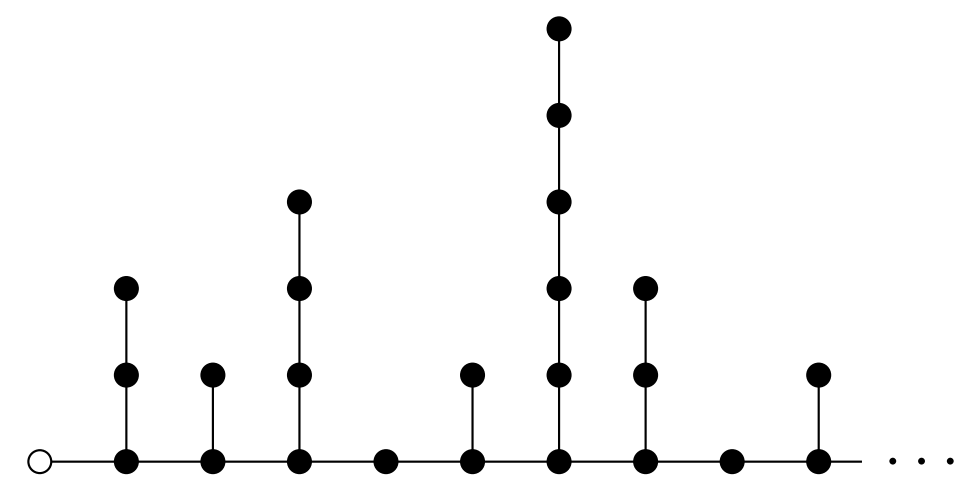

Fig. 1: A comb.

teeth of infinite length everywhere we shall denote the generating functions for first return probabilities to $r$ by $P_{\infty}(x)$ and $P_{*}(x)$, respectively. These may easily be calculated, e.g. by making use of the following elementary result, whose proof is left to the reader.

Lemma 1 Let $G_{1}, \ldots, G_{n}$ be $n$ disjoint, connected graphs with roots $r_{1}, \ldots, r_{n}$, respectively, all of degree 1. Let $G$ be the graph obtained by identifying $r_{1}, \ldots, r_{n}$ with a common vertex s and, in addition, attaching a single edge $(r, s)$ to the vertex $s$. With $r$ as the root of $G$ we then have

$$
P_{G}(x)=\frac{1-x}{1+n-\sum_{i=1}^{n} P_{G_{i}}(x)} .
$$


One finds

$$
P_{\infty}(x)=1-\sqrt{x} \quad \text { and } \quad P_{*}(x)=1-x^{1 / 4} \sqrt{1+\frac{5}{4} \sqrt{x}}+\frac{1}{2} \sqrt{x} .
$$

Further simple consequences of Lemma 1 applied to combs are (see [8] for details):

The monotonicity property: $P_{C}(x)$ is a monotonically decreasing function of each tooth length.

The rearrangement property: If two combs $C^{\prime}$ and $C^{\prime \prime}$ deviate by the interchange of two neighbouring teeth of different length, then the one with the shorter tooth closer to the root has the larger first return generating function.

A particular consequence of the monotonicity property is that

$$
P_{*}(x) \leq P_{C}(x) \leq P_{\infty}(x)
$$

for all combs $C$. From $(5)$ it then follows that

$$
1 \leq d_{s} \leq 3 / 2
$$

for any ensemble of combs. Below we provide examples showing that any value of $d_{s}$ in this range occurs.

We shall restrict attention to comb ensembles for which the tooth lengths are independently and identically distributed. An ensemble $(\mathcal{C}, \nu)$ is then uniquely specified by the tooth length probabilities $p_{\ell}, \ell \in$ $\{0,1,2, \ldots, \infty\}$. The following result is an extension of a result proven in [8] and shows, in particular, that if infinite teeth occur, then $d_{s}=3 / 2$.

Theorem 1 Assume $p_{\infty} \equiv p>0$. Then for almost every comb $C$ there exists a constant $\Lambda_{C}>0$ such that

$$
x^{-1 / 4} \leq Q_{C}(x) \leq \Lambda_{C} x^{-1 / 4}|\log x|^{1 / 2},
$$

and there exists a constant $\Lambda>0$ such that

$$
x^{-1 / 4} \leq Q(x) \leq \Lambda x^{-1 / 4}|\log x|^{1 / 2}
$$

both valid for $0<x<1 / 2$.

Proof: The lower bounds follow immediately from (3) and the lower bound in (6). For the upper bounds it is sufficient to consider the case where $p_{\ell}=0$ for $\ell \neq 0, \infty$, by the monotonicity property. In this case, we denote by $L_{i}, i=1,2, \ldots$, the distance along the spine between the $i$ 'th and the $(i+1)$ 'st tooth in a comb and by $L_{0}$ the distance from the root to the first tooth. Clearly, the $L_{i}$ are i.i.d. random variables, and for fixed integers $k, L>0$ we have

$$
\nu\left(\left\{L_{i} \leq L \text { for } i=0,1, \ldots, k-1\right\}\right)=\left(1-q^{L}\right)^{k},
$$

where $q=1-p$. We choose $L$ and $k$ as functions of $x$ according to

$$
L(x)=[a|\log x|]+1 \quad \text { and } \quad k(x)=\left[x^{-1 / 2-\delta}\right]
$$

where $a, \delta$ are positive constants to be further specified below, and [.] denotes integer part. Defining the event

$$
\mathcal{A}_{a, \delta}(x) \equiv\left\{L_{i} \leq L(x) \text { for all } i=0,1, \ldots, k(x)\right\},
$$

we have

$$
\nu\left(\mathcal{A}_{a, \delta}(x)\right) \geq 1-\frac{|\log p|}{q} x^{a|\log q|-1 / 2-\delta} .
$$

Choosing $a, \delta$ such that $a|\log q|-1 / 2-\delta>1 / 4$, we obtain

$$
\nu\left(\mathcal{A}_{a, \delta}(x)\right) \geq 1-o\left(x^{1 / 4}|\log x|^{1 / 2}\right) .
$$

Hence, for the chosen values of $a, \delta$ it follows from (3) and (6) that

$$
Q(x)=\int_{\mathcal{A}_{a, \delta}(x)} Q_{C}(x) d \nu(C)+o\left(x^{-1 / 4}|\log x|^{1 / 2}\right) .
$$


Consider now $C \in \mathcal{A}_{a, \delta}(x)$ and let $C^{\prime}$ be the comb obtained by deleting all teeth except the first $k(x)$ ones and then shifting these suitably away from the root such that they have constant spacing $L(x)$. From the monotonicity and rearrangement properties it follows that

$$
P_{C}(x) \leq P_{C^{\prime}}(x)=P_{C^{\prime}}^{(1)}(x)+P_{C^{\prime}}^{(2)}(x)
$$

where we have introduced the notation $P_{C}^{(1)}(x)$ for the contribution to $P_{C^{\prime}}(x)$ coming from walks $\omega$ that do not pass through the vertex $\left[x^{-1 / 2-\delta}\right]$ on the spine, and $P_{C^{\prime}}^{(2)}(x)$ for the remaining contribution. For the latter the bound

$$
P_{C^{\prime}}^{(2)}(x) \leq c_{1} \exp \left(-c_{2} x^{-\delta / 2}\right)
$$

holds with positive constants $c_{1}, c_{2}$ independent of $C^{\prime}$. This inequality is easy to verify for the case $C^{\prime}=$ $N_{\infty}$ and follows for arbitrary $C^{\prime}$ by a simple generalisation of the upper bound in (6) to the generating function for transition probabilities for walks with endpoint different from the root, see [8] Section 2.5 for details. On the other hand, we have

$$
P_{C^{\prime}}^{(1)}(x)=P_{* L}^{(1)}(x) \leq P_{* L}(x)
$$

where $* L$ denotes the comb with infinite teeth of spacing $L$ along the spine. Here $P_{* L}(x)$ can be calculated by using Lemma 1 . The result is (see [8], Appendix 2)

$$
P_{* L}(x)=1-\frac{x^{1 / 4}}{\sqrt{L}}+O(\sqrt{x}) .
$$

With our choice of $L$ we get from the preceding four equations that

$$
P_{C}(x) \leq 1-\frac{x^{1 / 4}}{\sqrt{L(x)}}+O(\sqrt{x}) \leq 1-\left(a+\frac{1}{\log 2}\right)^{-1 / 2} x^{1 / 4}|\log x|^{-1 / 2}+O(\sqrt{x})
$$

valid uniformly for $C \in \mathcal{A}_{a, \delta}(x)$ and $0<x<1 / 2$. Using this estimate for fixed $a, \delta$ together with 3 in (10) we obtain (8).

To obtain (7), we fix $A, \delta>0$ such that $A|\log q|-1 / 2-\delta>1$, and set

$$
\mathcal{A}_{a} \equiv \bigcap_{n \geq 3} \mathcal{A}_{a, \delta}(1 / n) \quad \text { for } a \geq A
$$

By (9) we then have

$$
\begin{aligned}
\nu\left(\mathcal{A}_{a}\right) & \geq 1-\frac{|\log p|}{q} \sum_{n \geq 3} n^{-(a|\log q|-1 / 2-\delta)} \\
& \geq 1-\frac{|\log p|}{q} \int_{2}^{\infty} x^{1 / 2+\delta-a|\log q|} d x \\
& =1-\frac{|\log p| 2^{3 / 2+\delta}}{q(a|\log q|-3 / 2-\delta)} 2^{-a|\log q|} .
\end{aligned}
$$

Clearly, $\mathcal{A}_{a}$ increases with $a$, and it follows from this inequality that the set

$$
\mathcal{A} \equiv \bigcup_{a \geq A} \mathcal{A}_{a}
$$

has measure 1 , and for every $C \in \mathcal{A}$ there exists by (11) a constant $\Delta_{C}>0$ such that

$$
P_{C}(1 / n) \leq 1-\Delta_{C}(1 / n)^{1 / 4}|\log 1 / n|^{-1 / 2}, \quad n=3,4,5, \ldots
$$

Since $P_{C}(x)$ is a decreasing function of $x$, this together with (3) evidently implies (7) for a suitable constant $\Lambda_{C}>0$, and the theorem is proven.

The next result concerns combs with finite teeth only, and was conjectured in [12] on the basis of mean field arguments. 
Theorem 2 Let $a>1$ and $p_{\ell}=c_{a} \ell^{-a}$, where $c_{a}$ is a normalisation factor such that $\sum_{\ell=1}^{\infty} p_{\ell}=1$. Then the spectral dimension for the comb ensemble defined by $\left(p_{\ell}\right)$ is given by

$$
d_{s}=\left\{\begin{array}{cc}
(4-a) / 2 & \text { if } 1<a \leq 2 \\
3 / 2 & \text { if } a>2 .
\end{array}\right.
$$

The required upper bounds, analogous to that of [8] (and in fact likewise an analogue of the upper bound in (7)), can be obtained by arguments quite similar to those of the preceding proof. The lower bounds for $1<a<2$, on the other hand, require different methods. We refer the interested reader to [8], Section 4.

\section{Generic random trees}

In this section we introduce what we call generic ensembles of trees obtained as limits of certain GaltonWatson processes conditioned to have a fixed total number of progeny.

Let $\Gamma$ be the set of all planar rooted trees, finite or infinite, such that the root, $r$, is of order (or valency) 1. If $\tau \in \Gamma$ is finite we let $|\tau|$ denote its size, i.e. the number of links in $\tau$, and the subset of $\Gamma$ consisting of trees of fixed size $N$ will be called $\Gamma_{N}$. Given a tree $\tau \in \Gamma$, the ball $B_{R}(\tau)$ of radius $R$ around the root is the subgraph of $\tau$ spanned by the vertices whose graph distance from $r$ is less than or equal to $R$. It is useful to define the distance $d_{\Gamma}\left(\tau, \tau^{\prime}\right)$ between two trees $\tau, \tau^{\prime}$ as $(R+1)^{-1}$, where $R$ is the radius of the largest ball around $r$ common to $\tau$ and $\tau^{\prime}$. We shall view $\Gamma$ as a metric space with metric $d_{\Gamma}$, see [7] for some of its properties.

Given a Galton-Watson process with offspring probabilities $p_{n}, n=0,1,2, \ldots$, and with an ancestor conditioned to have exactly one offspring, let $Z_{N}$ denote the probability that the total number of progeny equals $N$,

$$
Z_{N}=\sum_{\tau \in \Gamma_{N}} \prod_{i \in \tau \backslash r} p_{\sigma_{i}-1}
$$

where the product is over all vertices in $\tau$ different from the root $r$, and $\sigma_{i}$ denotes the degree of vertex $i$. We assume as usual $p_{0}>0$ and that $p_{n}>0$ for some $n \geq 2$. Under these assumptions the generating function $g$ for the offspring probabilities,

$$
g(s)=\sum_{n=0}^{\infty} p_{n} s^{n}
$$

is strictly increasing and strictly convex on the interval $[0, \rho[$, where $\rho$ is the radius of convergence for the series (13), which we naturally assume is non-vanishing.

It is well known, see e.g. [3] Section 2.5, that the generating function for the total number of progeny,

$$
Z(x)=\sum_{N=1} Z_{N} x^{N}
$$

satisfies

$$
Z(x)=x g(Z(x)) .
$$

Indeed, eq. 15 determines $Z(x)$ uniquely as an analytic function of $x$, vanishing at $x=0$. Letting $x_{0}$ denote the radius of convergence of the series (14), the limit

$$
Z_{0}=\lim _{x \nearrow x_{0}} Z(x)
$$

is finite and $\leq \rho$. In the following we shall restrict attention to the generic case

$$
Z_{0}<\rho,
$$

omitting the case $Z_{0}=\rho$ from further consideration. Then $Z_{0}$ is determined by the equation

$$
Z_{0} g^{\prime}\left(Z_{0}\right)=g\left(Z_{0}\right) \text {. }
$$

By Taylor expanding $g$ around $Z_{0}$ and using eqs. (15) and (17) one obtains the well known singular behaviour of $Z$ at $x_{0}$,

$$
Z(x)=Z_{0}-\sqrt{\frac{2 g\left(Z_{0}\right)}{x_{0} g^{\prime \prime}\left(Z_{0}\right)}} \sqrt{x_{0}-x}+O\left(x_{0}-x\right) .
$$

We shall need the following result on the asymptotic behaviour of $Z_{N}$, the proof of which can be found in [10] Section VI.5 and VII.2. 
Lemma 2 Assuming (16) the asymptotics of $Z_{N}$ is given by

$$
Z_{N}=\sqrt{\frac{g\left(Z_{0}\right)}{2 \pi g^{\prime \prime}\left(Z_{0}\right)}} N^{-\frac{3}{2}} x_{0}^{-N}\left(1+O\left(N^{-1}\right)\right),
$$

if the integers $n$ such that $p_{n} \neq 0$ have no common divisor $>1$. Otherwise, if $d \geq 2$ denotes their largest common divisor, we have

$$
Z_{N}=d \sqrt{\frac{g\left(Z_{0}\right)}{2 \pi g^{\prime \prime}\left(Z_{0}\right)}} N^{-\frac{3}{2}} x_{0}^{-N}\left(1+O\left(N^{-1}\right)\right),
$$

if $N \equiv 1 \bmod d$, and $Z_{N}=0$ otherwise.

Now let the probability measure $\nu_{N}$ be defined on $\Gamma_{N}$ by

$$
\nu_{N}(\tau)=Z_{N}^{-1} \prod_{i \in \tau \backslash r} p_{\sigma_{i}-1}, \quad \tau \in \Gamma_{N},
$$

provided $Z_{N} \neq 0$. Using the preceding lemma, the existence of the weak limit of the sequence $\left(\nu_{N}\right)$ on $\Gamma$ can be proven by a minor modification of the arguments in [7], where the existence was proven for the so-called uniform measure corresponding to $p_{n}=2^{-n-1}, n \geq 0$, with $\rho=2$ and $Z_{0}=1$. We state the result in the following proposition.

Proposition 1 Considering $\nu_{N}$ as a probability measure on $\Gamma$, with the same assumptions as in Lemma 1 , the weak limit

$$
\nu=\lim _{N \rightarrow \infty} \nu_{N}
$$

exists and is a probability measure on $\Gamma$ supported on the subset $\Gamma_{\infty}$ of infinite trees. 16.

We call the ensembles $(\Gamma, \nu)$ so obtained generic ensembles of infinite trees, referring back to assumption

As for the uniform measure, the measure $\nu$ can be described in simple terms. First, $\nu$ is supported on the subset of trees with a single spine (or backbone). Such a tree is given by an infinite linear chain starting at the root $r$, called the spine, whose vertices we shall denote by $s_{0}=r, s_{1}, s_{2}, \ldots$, ordered by increasing distance from the root. To each $s_{n}, n \geq 1$, are attached a finite number of branches, i.e. finite trees in $\Gamma$, by identifying their roots with $s_{n}$. If $s_{n}$ is of degree $\sigma$ there are $\sigma-2$ branches. Let $T_{1}^{\prime}, \ldots, T_{k}^{\prime}$ denote those to the left (relative to the direction from $r$ along the spine) and $T_{1}^{\prime \prime}, \ldots, T_{\ell}^{\prime \prime}$ those to the right, ordered clockwise around $s_{n}$. Then the probability that $s_{n}$ has $k \geq 0$ left branches and $\ell \geq 0$ right branches equals

$$
\varphi(k, \ell)=x_{0} p_{k+\ell+1} Z_{0}^{k+\ell},
$$

independently of $n$. Finally, given $k, \ell$, the $k+\ell$ branches at $s_{n}$ are independently distributed according to the Galton-Watson process with offspring probabilities given by

$$
r_{n}=x_{0} p_{n} Z_{0}^{n-1}, \quad n=0,1,2, \ldots
$$

Furthermore, this process is critical as a consequence of (17), i.e.

$$
\sum_{n=1}^{\infty} n r_{n}=1
$$

In the following we let $f$ denote the generating function for the offspring probabilities given by (22),

$$
f(s)=\sum_{n=0}^{\infty} r_{n} s^{n}=x_{0} \sum_{n=0}^{\infty} p_{n} Z_{0}^{n-1} s^{n}=x_{0} Z_{0}^{-1} g\left(Z_{0} s\right) .
$$

such that

$$
f(1)=1 \quad \text { and } \quad f^{\prime}(1)=1,
$$

and the assumption (16) is equivalent to assuming $f$ to be analytic in a neighbourhood of the unit disc.

The next theorem establishes that the spectral dimension of the generic ensembles introduced above equals $4 / 3$. 
Theorem 3 For any generic ensemble of infinite trees there exist constants $\underline{c}, \bar{c}>0$ such that

$$
\underline{c} x^{-1 / 3} \leq Q(x) \leq \bar{c} x^{-1 / 3} .
$$

Proof: We confine ourselves to explaining the main ingredients of the proof. Detailed arguments can be found in [9]. Thus, let $(\Gamma, \nu)$ be a generic ensemble.

Lower bound on $Q(x)$.

The following two inequalities are easily proven by induction, using Lemma 1 .

i) For any finite tree $T \in \Gamma$ and $0<x<1$

$$
P_{T}(x) \geq 1-|T| x
$$

ii) For any tree $\tau \in \Gamma$ with one infinite spine and $L \geq 1$

$$
P_{\tau}(x) \geq 1-\frac{1}{L}-L x-\sum_{T \subset \tau}^{L}\left(1-P_{T}(x)\right),
$$

where $\sum_{T \subset \tau}^{L}$ denotes the sum over all (finite) branches $T$ of $\tau$ attached to vertices on the spine at distance $\leq L$ from the root.

Let $\tau \in \Gamma$ be a tree with a single spine and let $s_{n}$ be any vertex on the spine different from $r$. Since each branch at $s_{n}$ is a critical Galton-Watson tree, it is well known (see e.g. [11] Section I.10) that the probability that it has height $>R$ asymptotically equals

$$
\frac{2}{f^{\prime \prime}(1) R}, \quad \text { for } R \text { large . }
$$

Hence, the conditional probability $c_{R}$ that at least one branch at $s_{n}$ has height $>R$, given that $k+\ell$ branches are attached at $s_{n}$, fulfills

$$
c_{R} \leq(k+\ell) \frac{c^{\prime}}{R}
$$

for some positive constant $c^{\prime}$. According to 21) the $\nu$-probability that at least one branch at $s_{n}$ has height $>R$ is hence bounded from above by

$$
\frac{c^{\prime}}{R} \sum_{k, \ell \geq 0}(k+\ell) \varphi(k, \ell)=x_{0} \frac{c^{\prime}}{R} \sum_{i=0}^{\infty} i(i+1) p_{i+1} Z_{0}^{i}=\frac{c^{\prime \prime}}{R}
$$

where $c^{\prime \prime}$ is a positive constant, since the last sum is convergent by assumption (16).

By independence of the distribution of branches attached to different vertices on the spine we conclude that the $\nu$-probability of the event $\mathcal{A}_{R}$, that all branches attached to the first $R$ vertices $s_{1}, \ldots, s_{R}$ on the spine have height $\leq R$, satisfies

$$
\nu\left(\mathcal{A}_{R}\right)=\left(1-c_{R}\right)^{R} \geq\left(1-\frac{c^{\prime}}{R}\right)^{R} \geq c^{\prime \prime \prime}
$$

where $c^{\prime \prime \prime}$ is a positive constant, when $R$ is sufficiently large. Denoting by $\langle\cdot\rangle_{R}$ the expectation w.r.t. $\nu$ conditioned on the event $\mathcal{A}_{R}$, we get by i) and ii) above and Jensen's inequality that

$$
\begin{aligned}
Q(x) & \geq c^{\prime \prime \prime}\left\langle\left(1-P_{\tau}(x)\right)^{-1}\right\rangle_{R} \\
& \geq c^{\prime \prime \prime}\left\langle\left(\frac{1}{R}+R x+\sum_{T \subset \tau}^{R} x|T|\right)^{-1}\right\rangle_{R} \\
& \geq c^{\prime \prime \prime}\left(\frac{1}{R}+R x+x\left\langle\sum_{T \subset \tau}^{R}|T|\right\rangle_{R}\right)^{-1} .
\end{aligned}
$$


Noting that the distributions of branches are identical at all vertices on the spine, given by (21) and the offspring probabilities 22, we have

$$
\begin{aligned}
\left\langle\sum_{T \subset \tau}^{R}|T|\right\rangle_{R} & =\left\langle\sum_{T \subset \tau}^{R}\left|B_{R}(T)\right|\right\rangle_{R} \\
& \leq\left(1-c_{R}\right)^{-1}\left\langle\sum_{T \subset \tau}^{R}\left|B_{R}(T)\right|\right\rangle_{\nu} \\
& =\left(1-c_{R}\right)^{-1} R \sum_{k, \ell \geq 0} \varphi(k, \ell)(k+\ell)\left\langle\left|B_{R}\right|\right\rangle_{\mu} \\
& =\left(1-c_{R}\right)^{-1} R(R+1) \sum_{k, \ell \geq 0}(k+\ell) \varphi(k, \ell) \\
& =\frac{c^{\prime \prime}}{c^{\prime}\left(1-c_{R}\right)} R(R+1),
\end{aligned}
$$

where we have used the elementary result (see e.g. [11] Section I.5) that for a critical Galton-Watson process the average value of $\left|B_{R}(T)\right|$ equals $R+1$.

Inserting this estimate into 27 and recalling that $c_{R} \rightarrow 0$ as $R \rightarrow 0$ we deduce

$$
Q(x) \geq c^{\prime \prime \prime}\left(\frac{1}{R}+R x+\frac{2 c^{\prime}}{c^{\prime \prime}} x R^{2}\right)^{-1} .
$$

for $R$ large enough. Finally, choosing $R=\left[x^{-1 / 3}\right]$ now yields

$$
Q(x) \geq \underline{c} x^{-1 / 3}
$$

for a suitable constant $\underline{c}>0$, as claimed.

Upper bound on $Q(x)$.

This is based on the inequality

$$
Q_{\tau}(x) \leq \frac{2}{x\left|B_{R}(\tau)\right|}+R
$$

valid for all trees $\tau \in \Gamma$ and all $R \geq 1$. An analogue of this inequality was established in [5], Section 4, for the return probability for the continuous time random walk. The arguments can be adapted to the discrete time random walk and the generating function setting used here. An alternative and more straight forward proof is given in [9].

Furthermore, one proves that

$$
\left\langle\left|B_{R}\right|^{-1}\right\rangle_{\nu} \leq c R^{-2}
$$

for all $R \geq 1$ by a rather straightforward argument. This result may be well known. Note in passing that the identity

$$
\left\langle\left|B_{R}\right|\right\rangle_{\nu}=\frac{1}{2} f^{\prime \prime}(1) R(R-1)+R
$$

is easy to establish and expresses the fact that the so-called Hausdorff dimension of the generic ensembles is 2 .

Taking the $\nu$-average on both sides of (28) and using 29] gives

$$
Q(x) \leq \frac{2 c}{x R^{2}}+R
$$

for all $R \geq 1$. Setting $R=\left[x^{-1 / 3}\right]$, the two terms are of same order of magnitude $x^{-1 / 3}$ for $x$ small, that is

$$
Q(x) \leq \bar{c} x^{-1 / 3}
$$

for a suitable constant $\bar{c}>0$. 
Remark 1 The results of this section also apply to the case where the ancestor has any fixed number $m \geq 1$ of offspring. Denoting by $\Gamma^{(m)}$ the set of planar trees with a distinguished root link $\left(r, r^{\prime}\right)$ and such that $r$ has degree $m$, we define the numbers $Z_{N}^{(m)}, N \geq m$, by the righthand side of 12 with $\Gamma_{N}$ replaced by $\Gamma_{N}^{(m)}=\left\{\tau \in \Gamma^{(m)}:|\tau|=N\right\}$. The corresponding generating function $Z^{(m)}(x)$ is then given by $Z^{(m)}(x)=Z(x)^{m}$, where $Z(x)$ is as in 18 . This relation implies an immediate generalisation of Lemma 2 and also the existence of a probability measure $\nu^{(m)}$ supported on the subset of $\Gamma^{(m)}$ consisting of trees with one infinite spine originating from $r$ and with a similar characterisation as the measure $\nu$. In particular, the (finite) branches have the same probability distribution as in the $m=1$ case and the $m$ branches (including the infinite one) originating from $r$ have equal probabilities of being infinite. On the basis of this observation the proof of Theorem 3 can be carried through with minor modifications to yield the same upper and lower bounds 26) for the generating function $Q^{(m)}(x)$ for return probabilities to the root $r$.

Similar remarks apply to the case where the ancestor is subject to the same offspring probability distribution as the descendants.

Remark 2 In [5] the asymptotic behaviour (1) with $d_{s}=4 / 3$ was established almost surely up to logarithmic factors for the return probabilities for the continuous time random walk on the incipient infinite percolation cluster on a Cayley tree. It seems that the methods used there also apply to the generic trees considered in this article to obtain similar results. The method of proof, however, in particular the part relating to the upper bound, is more involved than the proof of 26 given here.

\section{References}

[1] S. Alexander and R. Orbach, Density of states on fractals: ”fractons”, J. Physique Lett. 43 (1982) L625-L631.

[2] J. Ambjørn, D. Boulatov, J. L. Nielsen, J. Rolf and Y. Watabiki, The spectral dimension of 2D quantum gravity, JHEP 9802 (1998) 010

[3] J. Ambjørn, B. Durhuus and T. Jonsson, Quantum geometry: a statistical field theory approach, Cambridge University Press, Cambridge (1997)

[4] D. ben-Avraham and S. Havlin, Diffusion and reactions in fractals and disordered systems, Cambridge University Press, Cambridge, (2000)

[5] M. T. Barlow and T. Kumagai, Random walk on the incipient infinite cluster on trees, Preprint 2005, math.PR/0503118.

[6] C. Destri and L. Donetti, The spectral dimension of random trees, J. Phys. A 35 (2002) 9499-9516

[7] B. Durhuus, Probabilistic aspects of infinite trees and surfaces, Act. Phys. Pol. 34 (2003) 4795-4811

[8] B. Durhuus, T. Jonsson and J. Wheater, Random walks on combs, J. Phys. A A39 (2006) 1009-1038

[9] B. Durhuus, T. Jonsson and J. Wheater, The spectral dimension of generic trees, Preprint 2006.

[10] Ph. Flajolet and R. Sedgewick, Analytic Combinatorics, Online book available at http://algo.inria.fr/ flajolet/Publications/books.html

[11] T. E. Harris, The theory of branching processes, Dover Publications, Inc., New York, 2002.

[12] S. Havlin, J. E. Kiefer and G. H. Weiss, Anomalous diffusion on a random comblike structure, Phys. Rev. A 36 (1987) 1403-1408

[13] T. Jonsson and J. Wheater, The spectral dimension of the branched polymer phase of two-dimensional quantum gravity, Nucl. Phys. B 515 (1998) 549-574 
